\title{
An Inventory Model with Exponential Demand Rate, Finite Production Rate and Shortages
}

\author{
R. Begum ${ }^{1}$, S. K. Sahu ${ }^{2}$, and R. R. Sahoo ${ }^{3}$ \\ ${ }^{1}$ Department of Mathematics, Padmanava College of Engineering, Rourkela, Orissa-769002, India \\ ${ }^{2}$ National Institute of Financial Management, Faridabad, Hariyana-121001, India \\ ${ }^{3}$ Department of Physics, Synergy Institute of Technology, Bhubaneswar, Orissa-754001, India
}

Received 11 February 2009, accepted in revised form 15 August 2009

\begin{abstract}
The paper contains an order-level inventory model having the demand rate to be a function of time. Here shortages are allowed and completely backlogged. An optimal model is developed by considering exponential demand which minimizes the total average cost. Numerical examples are used to illustrate the developed model. Sensitivity analysis of the optimal solution with respect to major parameters is carried out.
\end{abstract}

Keywords: Inventory; EOQ; Finite production; Shortages; Exponential demand.

(c) 2009 JSR Publications. ISSN: 2070-0237 (Print); 2070-0245 (Online). All rights reserved.

DOI: $10.3329 /$ jsr.v1i3.2054

J. Sci. Res. 1 (3), 473-483 (2009)

\section{Introduction}

Demand is the major factor in inventory management. In inventory models, four types of demand are basically assumed i.e. constant demand, time-dependent demand, probabilistic demand and stock-dependent demand. Inventory models with stock-dependent demand are getting more attracted in present situation. Therefore, many authors studied these models in depth. Gupta and Vrat [1] assumed demand to be dependent on initial stock levels. On the other hand, Baker and Urban [2] considered the on-hand inventory demand in polynomial form. The constant demand is valid only when the phase of the product life cycle is matured for finite periods of time. But the assumption of this constant demand rate is not always applicable to other inventory items like fashionable clothes, electronic equipments and delicious foods due to the reason of variation in demand rate.

In the competitive market, the demand of some product may increase due to the consumer's preference on some eye-catching product. Therefore, the demand of the product at the time of its growth and the phase of declination may be approached by continuous-time-dependent function. These continuous-time-dependent functions may be

\footnotetext{
${ }^{3}$ Corresponding author: rakesh.s24@gmail.com
} 
a function of exponential or linear type. Ritchie [3] discussed the solution of a linear increasing time-dependent demand, which is obtained by Donaldson [4]. Silver and Meal [5] developed a model for deterministic time-varying demand, which also gives an approximate solution procedure termed as Silver-Meal Heuristic. Donaldson [4] discussed the policy for a linear, time-dependent demand with no shortages. Deb and Chaudhuri [6] reconsidered the extension of Donaldson [4] model. Dave [7] and Goyal et al. [8] derived the optimal method for different replenishment policy by allowing shortages. Goswami and Chaudhuri [9, 23] developed an EOQ model by assuming a linear trend demand, finite rate of replenishment with shortages. Another EOQ models for linear trend in demand was adopted by Dave and Patel [10] and Sachan [11]. Further, deteriorating items with exponential demand developed by Aggarwal and Bahari-Kashani [12] and Wee [13]. Raffat et al. [14] and Mak [15] considered EOQ models depending upon deterioration and other different assumptions like instantaneous or finite production rate. Ouyang et al. [20] proposed an EOQ model for deteriorating items with exponentially decreasing demand where shortages are allowed and partially backordered. Here, the backlogging rate is variable and dependent for the next replenishment on the waiting time. Bhunia and Maiti [22] developed the model in which the production rate is variable. They presented the model where shortages are not allowed and the production rate depends on either the onhand inventory or on the demand.

Depending upon the production rate, Su et al. [19] presented a production inventory model for deteriorating items with an exponentially declining demand over a fixed time horizon. In this model, the production rate depends on demand. In that paper, shortages are allowed and completely backlogged. Ghosh et al. [16] developed a model with timedependent demand, finite production rate and shortages by assuming a two- parameter Weibull demand rate. Here shortages are allowed and completely backlogged. Balkhi and Benkeherouf [17] developed a model by taking the consideration of fixed production schedule for deteriorating items in which demand and the production are allowed to vary with time in an arbitrary way at a constant rate of deterioration. Other researchers like Wee and Law [18] assumed a deterministic model and considered deteriorating items with price-dependent demand rate, a time-varying deterioration rate and finite production rate with time value of money over a fix time horizon. Hollter and Mak [21] developed inventory replenishment policies for deteriorating items. They considered replenishment problems with declining demand. Again, Aggarwal and Bahari-Kashani [12] developed a model assuming flexible production rate for deteriorating items.

In the present article, we have assumed an inventory model for items with exponential demand. The production rate is finite and proportional to the demand rate. The timedependent demand rate increases exponentially or decreases depending upon the shape parameter. Here shortages are allowed and completely backlogged. Numerical examples have considered for illustrating the developed model. The development of this model is to minimize the total average cost. Sensitivity analysis is carried out by taking the account of major parameters. Finally, the total average cost and some computational procedure of a few important results have been shown in Appendices A, B and C. 


\section{Assumptions and Notations}

The mathematical model of the inventory problem is developed on the following assumptions:

(a) The demand rate $R$ at any time is given by $R(t)=\alpha e^{\beta t}, \alpha, \beta>0$

(b) The production rate $K(t)$, at any instant depends on the demand is a constant and is given by $K(t)=\lambda R(t), \lambda>1$ and $K(t)>R(t)$.

(c) The lead-time is zero.

(d) The on-hand inventory does not deteriorate with time.

(e) Shortages are allowed and backlogged completely.

The notations used: $C_{1}$ is the carrying cost per unit time, $C_{2}$ is the shortage cost per unit time, $C_{3}$ is the set up cost per production and $C$ is the total average cost for a production cycle. All these costs $C_{1}, C_{2}, C_{3}$ are fixed and known at the time of production run.

\section{Mathematical Modeling and Solution}

Initially, the stock level is zero at time $t=0$. Again at $t=0$, the shortage starts and accumulates to the level $P$ at $t=t_{1}$. The production inventory level starts at $t=t_{1}$. At the instant of time, the production starts to clear the backlog by the time $t=t_{2}$. Then the production is stopped, the stock level attains a level $S$ at $t=t_{3}$. The inventory level becomes zero at time $t=t_{4}$. This decrease in level occurs due to the demand. After time $t_{4}$, the repetition of the inventory cycle occurs. The aim is to find out the optimum values of $t_{1}, t_{2}, t_{3}, t_{4}, S, P$ which minimize the total average cost $c$.

Let $Q(t)$ represents the instantaneous inventory level at any time $t\left(0 \leq t \leq t_{4}\right)$. The differential equations governing the instantaneous states of $Q(t)$ in the interval $\left(0, t_{4}\right)$ are as follows

$$
\begin{aligned}
& \frac{d Q}{d t}=-R, \quad 0 \leq t \leq t_{1} \\
& \frac{d Q}{d t}=K-R, \quad t_{1} \leq t \leq t_{2} \\
& \frac{d Q}{d t}=K-R, \quad t_{2} \leq t \leq t_{3} \\
& \frac{d Q}{d t}=-R, \quad t_{3} \leq t \leq t_{4}
\end{aligned}
$$

with the following boundary conditions

$$
Q(0)=0, Q\left(t_{1}\right)=-P, Q\left(t_{2}\right)=0, Q\left(t_{3}\right)=S \text { and } Q\left(t_{4}\right)=0 .
$$

Using the value of $R(t)=\alpha e^{\beta t}$ and $K(t)=\lambda R(t)$, the Eqs.(1)-(4) reduces to the form

$$
\begin{aligned}
& \frac{d Q}{d t}=-\alpha e^{\beta t}, 0 \leq t \leq t_{1} \\
& \frac{d Q}{d t}=(\lambda-1) \alpha e^{\beta t}, t_{1} \leq t \leq t_{2}
\end{aligned}
$$




$$
\begin{aligned}
& \frac{d Q}{d t}=(\lambda-1) \alpha e^{\beta t}, t_{2} \leq t \leq t_{3} \\
& \frac{d Q}{d t}=-\alpha e^{\beta t}, t_{3} \leq t \leq t_{4}
\end{aligned}
$$

and the boundary conditions are $Q(0)=0, Q\left(t_{1}\right)=-P, Q\left(t_{2}\right)=0, Q\left(t_{3}\right)=S$ and $Q\left(t_{4}\right)=0$. Solving the equations (6)-(9) and substituting the above boundary conditions, the solutions are as follows:

$$
\begin{aligned}
& Q(t)=\left(\frac{\alpha}{\beta}\right)\left[1-e^{\beta t}\right], 0 \leq t \leq t_{1} \\
& =(\lambda-1) \frac{\alpha}{\beta}\left[e^{\beta t}-e^{\beta t_{2}}\right], t_{1} \leq t \leq t_{2} \\
& =(\lambda-1) \frac{\alpha}{\beta}\left[e^{\beta t}-e^{\beta t_{2}}\right], t_{2} \leq t \leq t_{3} \\
& =\left(\frac{\alpha}{\beta}\right)\left[e^{\beta t_{4}}-e^{\beta t}\right], t_{3} \leq t \leq t_{4}
\end{aligned}
$$

Substituting the initial condition $Q\left(t_{1}\right)=-P$ in equation (10) and (11), we obtain (see Appendix A)

$$
t_{1}=\left(\frac{1}{\beta}\right) \log \left[\frac{1+(\lambda-1) e^{\beta t_{2}}}{\lambda}\right]
$$

Substituting the initial condition $Q\left(t_{3}\right)=S$ in equation (12) and (13), we obtain (see Appendix A)

$$
t_{3}=\left(\frac{1}{\beta}\right) \log \left[\frac{e^{\beta t_{4}}+(\lambda-1) e^{\beta t_{2}}}{\lambda}\right]
$$

The total average cost of the system is (see Appendix B)

$$
\begin{aligned}
C & =\frac{1}{t_{4}}\left[S C+H C+c_{3}\right] \\
= & \frac{1}{t_{4}}\left[\begin{array}{l}
c_{2}\left[-\frac{\alpha}{\beta}\left\{t_{1}-\frac{e^{\beta t_{1}}-1}{\beta}\right\}-(\lambda-1)\left(\frac{\alpha}{\beta}\right)\left\{\frac{e^{\beta t_{2}}-e^{\beta t_{1}}}{\beta}-e^{\beta t_{2}}\left(t_{2}-t_{1}\right)\right\}\right]+ \\
\left.c_{1}\left[(\lambda-1)\left(\frac{\alpha}{\beta}\right)\left\{\frac{e^{\beta t_{3}}-e^{\beta t_{2}}}{\beta}-e^{\beta t_{2}}\left(t_{3}-t_{2}\right)\right\}+\left(\frac{\alpha}{\beta}\right)\left\{e^{\beta t_{4}}\left(t_{4}-t_{3}\right)-\frac{e^{\beta t_{4}}-e^{\beta t_{3}}}{\beta}\right\}\right]+c_{3}\right]
\end{array}\right.
\end{aligned}
$$

Again substituting the values of $t_{1}$ from equation (14) and $t_{3}$ from equation (15), the equation (16), i.e. $C$ is a function of two variables $t_{2}$ and $t_{4}$. Now our aim is to minimize $C$ using calculus. The optimum values of $t_{2}$ and $t_{4}$ for the minimum average cost $C$ are the solutions of the equations

$$
\frac{\partial C}{\partial t_{2}}=0 \text { and } \frac{\partial C}{\partial t_{4}}=0
$$

and 


$$
\frac{\partial^{2} C}{\partial t_{2}^{2}} \frac{\partial^{2} C}{\partial t_{4}^{2}}-\left(\frac{\partial^{2} C}{\partial t_{2} \partial t_{4}}\right)^{2}>0
$$

Therefore, Eqs. (17) can be written as,

$$
\frac{1}{\beta t_{4}}\left[e^{\beta t_{2}} \alpha(\lambda-1)\left\{\beta\left(c_{1}+c_{2}\right) t_{2}-c_{1} \log \left[\frac{e^{\beta t_{4}}+(\lambda-1) e^{\beta t_{2}}}{\lambda}\right]-c_{2} \log \left[\frac{1+(\lambda-1) e^{\beta t_{2}}}{\lambda}\right]\right\}\right]=0
$$

and

$$
\begin{aligned}
\frac{1}{\beta^{2} t_{4}^{2}}\left[-\beta^{2} c_{3}+\alpha\right. & c_{2}\left(\left(1+(\lambda-1) e^{\beta t_{2}}\right) \log \left[\frac{1+e^{\beta t_{2}}(\lambda-1)}{\lambda}\right]-e^{\beta t_{2}} \beta(\lambda-1) t_{2}\right) \\
& +\alpha c_{1}\left(-e^{\beta t_{2}}(\lambda-1)\left(-\log \left[\frac{e^{\beta t_{4}}+(\lambda-1) e^{\beta t_{2}}}{\lambda}\right]+\beta t_{2}\right)\right. \\
& \left.\left.+e^{\beta t_{4}}\left(-1+\beta t_{4}\right)\left(-\log \left[\frac{e^{\beta t_{4}}+(\lambda-1) e^{\beta t_{2}}}{\lambda}\right]+\beta t_{4}\right)\right)\right]=0
\end{aligned}
$$

\section{Numerical Analysis}

Let us consider an inventory system with the following data: $\alpha=100, \beta=1.5, \lambda=2.5$, $c_{1}=25, c_{2}=30, c_{3}=40$ in appropriate units. From equation (19) and (20), we get the following optimal values of times $t_{1}{ }^{*}=0.0778299, t_{2}{ }^{*}=0.125087, t_{3}^{*}=0.181796$, $t_{4}^{*}=0.258727$. Again substituting these optimum values of times $t_{1}{ }^{*}, t_{2}{ }^{*}, t_{3}^{*}$ and $t_{4}^{*}$ in Eq. (16), we obtain the optimum average cost $C^{*}=283.522$ and the optimum values of $P$ and $S$ calculated from (A1) and (A4) are $P^{*}=8.25551$ and $S^{*}=10.711$ respectively (see Appendix A). Depending on the parameter $\beta$, we get the following results as follows:

Example-1 $0<\beta<1$

Let $\beta=0.5$, we get the following optimal values of times $t_{1}{ }^{*}=0.082317, t_{2}{ }^{*}=0.135371$, $t_{3}^{*}=0.199033$ and $t_{4}^{*}=0.290888$. The optimum average cost is $C^{*}=265.578$ and the optimum values of $P$ and $S$ are $P^{*}=8.40345$ and $S^{*}=10.3824$ respectively. In this case, there is retarded growth in demand (see Appendix C).

Example-2 $1<\beta<2$

Let $\beta=1.75$, the optimal times are $t_{1}{ }^{*}=0.0769075, t_{2}{ }^{*}=0.122971, t_{3}^{*}=0.178247$, $t_{4}^{*}=0.252269$. The optimum average cost is $C^{*}=287.759$ and the optimum values of $P$ and $S$ are $P^{*}=8.23231$ and $S^{*}=10.796$ respectively. This is the case of accelerated growth in demand. (see Appendix C) 


\section{Example-3 $\beta=1$}

The optimal values are $t_{1}{ }^{*}=0.0798881, t_{2}{ }^{*}=0.129808, t_{3}^{*}=0.189712, t_{4}^{*}=0.273334$, $C^{*}=274.769, P^{*}=8.31658$ and $S^{*}=10.5438$ respectively.

\section{Example-4 $\beta>2$}

Let $\beta=3.25$; the optimal values are $t_{1}{ }^{*}=0.0723943, t_{2}{ }^{*}=0.112648, t_{3}^{*}=0.160952$, $t_{4}^{*}=0.221615, C^{*}=311.645, P^{*}=8.162$ and $S^{*}=11.3139$ respectively. This is the case of accelerated growth in demand. (see Appendix C)

\section{Example-5 $\beta=2$}

The optimal values are $t_{1}{ }^{*}=0.0760434, \quad t_{2}{ }^{*}=0.12099, \quad t_{3}^{*}=0.174926$, $t_{4}^{*}=0.246277, C^{*}=291.913, P^{*}=8.21306$ and $S^{*}=10.8817$ respectively.

It is numerically verified that all the examples considered here are satisfying the sufficient condition in equation (18).

\section{Sensitive Analysis}

We now study the effects of changes in the system parameters $\alpha, \beta, \lambda, c_{1}, c_{2}, c_{3}$ on the optimal times of inventory interval $t_{1}^{*}, t_{2}^{*}, t_{3}^{*}, t_{4}^{*}$, on optimum average cost for a production cycle $C^{*}$ and also on the optimum values of $S$ and $P$ respectively. The sensitivity analysis is performed by changing each of the parameter by $+50 \%,+20 \%,+10 \%,-10 \%,-20 \%$ and $-50 \%$ taking one parameter at a time and keeping the remaining parameters unchanged. The results are shown in Table 1 . On the basis of the results of Table 1, the following observations can be made:

(a) With increase the value of $\alpha$, the value of $t_{1}^{*}, t_{2}^{*}, t_{3}^{*}, t_{4}^{*}, P^{*}, s^{*}$ and the optimum cost $C^{*}$ decreases. The obtained results show that $t_{1}^{*}, t_{2}^{*}, t_{3}^{*}, t_{4}^{*}$ and $C^{*}$ are moderately sensitive to changes in the value of $\alpha$.

(b) $t_{1}^{*}, t_{2}^{*}, t_{3}^{*}, t_{4}^{*}$ and $P^{*}$ decrease with increase in the value of the parameter $\beta$. At the same instant, the optimum cost $C^{*}$ and $S^{*}$ increase with the increase of $\beta$.

(c) With the increase of value of parameter $\lambda$, the value of $t_{1}{ }^{*}$ increases, but $t_{2}^{*}, t_{3}^{*}, t_{4}^{*}$ decreases. $P^{*}, S^{*}$ and the optimum cost $C^{*}$ are moderately sensitive with $\lambda$.

(d) As the value of parameter $c_{1}$ increases, the value of $t_{1}^{*}, t_{2}^{*}, c^{*}$ and $P^{*}$ increase. Moreover, $t_{3}^{*}, t_{4}^{*}$ and $s^{*}$ decrease with increase in the value of $c_{1}$.

(e) $t_{1}^{*}, t_{2}^{*}, t_{3}^{*}, t_{4}^{*}, P^{*}$ and $C^{*}$ decreases with the increase the value of $c_{2}$. But $s^{*}$ increases with increase of the value of parameter $C_{2}$. Moreover, $P^{*}$ and $S^{*}$ is highly sensitive to change in the parameter $C_{3}$. That means with the increase of values of $c_{3}$, $t_{1}^{*}, t_{2}^{*}, t_{3}^{*}, t_{4}^{*}, P^{*}$ and $S^{*}$ increase and the optimum cost $C^{*}$ increases these are highly sensitive to the parameter $C_{3}$. 
Table 1 . Sensitivity analysis.

\begin{tabular}{|c|c|c|c|c|c|c|c|c|}
\hline \multirow{2}{*}{ Parameter } & \multirow{2}{*}{$\begin{array}{l}\% \\
\text { change }\end{array}$} & \multicolumn{7}{|c|}{$\%$ change in } \\
\hline & & $t_{1}{ }^{*}$ & $t_{2}{ }^{*}$ & $t_{3}{ }^{*}$ & $t_{4}{ }^{*}$ & $C^{*}$ & $P^{*}$ & $S^{*}$ \\
\hline \multirow{6}{*}{$\alpha$} & +50 & -17.2021 & -16.7115 & -16.4596 & -15.9102 & +20.4139 & +22.9336 & +21.4144 \\
\hline & +20 & -8.1166 & -7.8617 & -7.7306 & -7.4483 & +8.6748 & +9.7291 & +9.0934 \\
\hline & +10 & -4.3236 & -4.1826 & -4.1106 & -3.9552 & +4.4373 & +4.9736 & +4.6494 \\
\hline & -10 & +4.9936 & +4.8166 & +4.7256 & +4.5325 & -4.6684 & -5.2241 & -4.8884 \\
\hline & -20 & +10.855 & +10.450 & +10.2422 & +9.8049 & -9.6080 & -10.7404 & -10.0548 \\
\hline & -50 & +37.448 & +35.752 & +34.8808 & +33.1090 & -26.7005 & -29.7216 & -27.8874 \\
\hline \multirow{6}{*}{$\beta$} & +50 & -3.3390 & -4.7630 & -5.4951 & -6.9698 & +4.3982 & -0.7047 & +2.3966 \\
\hline & +20 & -1.4133 & -2.0169 & -2.3273 & -2.9730 & +1.7896 & -0.3317 & +0.9532 \\
\hline & +10 & -0.7209 & -1.0288 & -1.1875 & -1.5209 & +0.9001 & -0.1749 & +0.4752 \\
\hline & -10 & +0.7521 & +1.0736 & +1.2382 & +1.5947 & -0.9117 & +0.1952 & -0.4733 \\
\hline & -20 & +1.5379 & +2.1952 & +2.5380 & +3.2710 & -1.8358 & +0.4122 & -0.9420 \\
\hline & -50 & +4.1354 & +5.8998 & +6.8059 & +8.8707 & -4.6867 & +1.2202 & -2.3219 \\
\hline \multirow{6}{*}{$\lambda$} & +50 & +11.413 & -7.5491 & -17.2919 & -8.2260 & +9.6408 & +12.1741 & +8.8255 \\
\hline & +20 & +5.8447 & -4.0339 & -9.1091 & -4.3942 & +4.9417 & +6.2137 & +4.5429 \\
\hline & +10 & +3.2249 & -2.2720 & -5.0963 & -2.4748 & +2.7278 & +3.4232 & +2.5123 \\
\hline & -10 & -4.0725 & +3.0466 & +6.7036 & +3.3173 & -3.4501 & -4.3046 & -3.1939 \\
\hline & -20 & -9.3913 & +7.3572 & +15.9612 & +8.0092 & -7.9644 & -9.8958 & -7.4012 \\
\hline & -50 & -45.1570 & +52.230 & +102.2624 & +56.7401 & -38.7229 & -46.6049 & -36.9257 \\
\hline \multirow{6}{*}{$c_{1}$} & +50 & +11.160 & +10.742 & -0.9153 & -7.9933 & +9.4701 & +11.9017 & -25.9889 \\
\hline & +20 & +5.1616 & +4.9781 & -0.5588 & -3.8708 & +4.3834 & +5.4853 & -12.4360 \\
\hline & +10 & +2.7233 & +2.6285 & -0.3261 & -2.0825 & +2.3141 & +2.8899 & -6.65866 \\
\hline & -10 & -3.0655 & -2.9643 & +1.0621 & +2.4578 & +2.6075 & -3.2421 & +7.7751 \\
\hline & -20 & -6.5460 & -6.3371 & +0.4565 & +5.4072 & -5.5717 & -6.9092 & +17.0002 \\
\hline & -50 & -20.6684 & -20.1020 & +5.4027 & +19.4722 & -17.6458 & -21.6362 & +59.8917 \\
\hline \multirow{6}{*}{$c_{2}$} & +50 & -27.0859 & -26.4001 & -14.3925 & -6.8956 & +7.8932 & -28.2488 & +7.2355 \\
\hline & +20 & -13.0043 & -12.6160 & -6.9253 & -3.3602 & +3.7041 & -13.6741 & +3.4067 \\
\hline & +10 & -6.9712 & -6.7497 & -3.7157 & -1.8123 & +1.9659 & -7.3560 & +1.8112 \\
\hline & -10 & +8.1568 & +7.8593 & +4.3570 & +2.1524 & -2.2439 & +8.6835 & -2.0745 \\
\hline & -20 & +17.847 & +17.144 & +9.5458 & +4.7536 & -4.8313 & +19.1083 & -4.4767 \\
\hline & -50 & +62.770 & +59.471 & +33.7515 & +17.3947 & -15.7585 & +69.0034 & -14.7366 \\
\hline \multirow{6}{*}{$C_{3}$} & +50 & +20.539 & +19.712 & +19.2875 & +18.4055 & +24.9338 & +22.0251 & +23.7867 \\
\hline & +20 & +8.7900 & +8.4677 & +8.3016 & +7.9527 & +10.4859 & +9.3610 & +10.0410 \\
\hline & +10 & +4.5082 & +4.3489 & +4.2668 & +4.0931 & +5.3431 & +4.7891 & +5.1237 \\
\hline & -10 & -4.7692 & -4.6143 & -4.53530 & -4.3644 & -5.5741 & -5.0390 & -5.3627 \\
\hline & -20 & -9.8460 & -9.5421 & -9.3863 & -9.0493 & -11.4185 & -10.3722 & -11.0039 \\
\hline & -50 & -27.6618 & -26.9665 & -26.6094 & -25.8191 & -31.2021 & -28.8397 & -30.2611 \\
\hline
\end{tabular}

\section{Conclusion}

The demand of a product may increase with time due to the incoming of a new product, which may be technically good and attractive than the old one, and also the demand of the new product may decrease with time. The given model deals with exponential timedependent increasing demand. Here the rate of production depends on demand. Shortages 
are allowed and are completely backlogged. Numerical examples and its sensitivity analysis for parameters are considered to assess the solution procedure.

The present paper develops an algorithm to determine demand, which is increasing or decreasing exponentially with time. The proposed model is more sensitive with respect to the parameter $\beta$. As the value of $\beta$ decreases, the demand decreases and as the value of $\beta$ increases, the demand increases. Therefore this vary of demand is really seen in stock market. Inventory modelers considered demands so far was only two types of timedependent demand i.e. linear and Weibull demands. For the first case, the demand rate function is of the form $R(t)=a+b t,(a \geq 0, b \neq 0)$, which implies steady increase or decrease in demand, which may be rarely seen to occur in the real market. For the second case, the demand rate function is of the form $R(t)=\alpha t^{\beta-1},(\alpha>0, \beta \neq 0)$. The demand rate increases at $\beta>1$, decreases at $\beta<1$ and constant at $\beta=1$. The time-dependent demand function, which we have assumed here, is of the form $R(t)=\alpha e^{\beta t},(\alpha>0, \beta \neq 0)$. If we consider an example of real world, in which we may face such exponentially avail situation.

In real market situation, demand is unlikely increase at a rate, which is very high as exponential. Whenever some new attractive products launched in super market or some seasonal items happen in beginning of season like winter, the demand of that product or item is increasing depending upon the rate of purchase. This type of demand is quite appropriate for products like winter vegetables, fruits in the city of Himachal Pradesh and Jammu-Kashmir, Rourkela etc. As the season progress, the demand rate goes on increasing and gradually approaching a saturation level. Similarly, considering the case of spare parts of newly introduced aircrafts, computer etc. The demand rate reaches a level of maximum as the production rate is increased. It may be noticed that as $\beta$ increases, the nonlinearity of the time-dependence demand rate increases (see Appendix C). Similarly, the spare parts of obsolete aircrafts, computer chip undergoes decline in demand rate. Therefore an exponential demand is more realistic than other type of demand.

\section{Acknowledgments}

The authors are very much grateful to the referee for his constructive comments for the improvement of the paper.

Appendix A. Solution of $P$ and $S$

Applying the condition $Q\left(t_{1}\right)=-P$ in equation (10), we have

$$
P=\left(\frac{\alpha}{\beta}\right)\left[e^{\beta t_{1}}-1\right]
$$

Further applying the same condition $Q\left(t_{1}\right)=-P$ in equation (11), we have

$$
P=-(\lambda-1)\left(\frac{\alpha}{\beta}\right)\left[e^{\beta t_{1}}-e^{\beta t_{2}}\right]
$$

Now equating the two values of $P$ from Eqs. (A1) and (A2), we have 


$$
t_{1}=\left(\frac{1}{\beta}\right) \log \left[\frac{1+(\lambda-1) e^{\beta t_{2}}}{\lambda}\right]
$$

Applying the boundary condition $Q\left(t_{3}\right)=S$ in Eqs. (12) and (13), we have the following relations

$$
S=(\lambda-1)\left(\frac{\alpha}{\beta}\right)\left[e^{\beta t_{3}}-e^{\beta t_{2}}\right]
$$

and $S=\left(\frac{\alpha}{\beta}\right)\left[e^{\beta t_{4}}-e^{\beta t_{3}}\right]$

Equating the two values of $S$ from equations (A4) and (A5), we have

$$
t_{3}=\left(\frac{1}{\beta}\right) \log \left[\frac{e^{\beta t_{4}}+(\lambda-1) e^{\beta t_{2}}}{\lambda}\right]
$$

Appendix B. Total average cost

The total cost per cycle includes shortage cost, holding cost and set up cost. Therefore, the total average cost of the system is given by,

$$
\begin{aligned}
& C=\frac{1}{t_{4}}\left[S C+H C+C_{3}\right] \\
& =\frac{1}{t_{4}}\left\{C_{2}\left[\int_{0}^{t_{1}}-Q(t) d t+\int_{t_{1}}^{t_{12}}-Q(t) d t+\right]+c_{1}\left[\int_{t_{2}}^{t_{3}}(\lambda-1)\left(\frac{\alpha}{\beta}\right)\left(e^{\beta t}-e^{\beta t_{2}}\right) d t+\int_{t_{3}}^{t_{4}}\left(\frac{\alpha}{\beta}\right)\left(e^{\beta t_{4}}-e^{\beta t}\right) d t\right]+c_{3}\right\}
\end{aligned}
$$

Therefore,

$$
C=\frac{1}{t_{4}}\left[\begin{array}{l}
c_{2}\left[-\frac{\alpha}{\beta}\left\{t_{1}-\frac{e^{\beta t_{1}}-1}{\beta}\right\}-(\lambda-1)\left(\frac{\alpha}{\beta}\right)\left\{\frac{e^{\beta t_{2}}-e^{\beta t_{1}}}{\beta}-e^{\beta t_{2}}\left(t_{2}-t_{1}\right)\right\}\right]+ \\
\left.c_{1}\left[(\lambda-1)\left(\frac{\alpha}{\beta}\right)\left\{\frac{e^{\beta t_{3}}-e^{\beta t_{2}}}{\beta}-e^{\beta t_{2}}\left(t_{3}-t_{2}\right)\right\}+\left(\frac{\alpha}{\beta}\right)\left\{e^{\beta t_{4}}\left(t_{4}-t_{3}\right)-\frac{e^{\beta t_{4}}-e^{\beta t_{3}}}{\beta}\right\}\right]+c_{3}\right]
\end{array}\right.
$$

Appendix C. Economic advantages of demand function

The demand rate function is $R(t)=\alpha e^{\beta t},(\alpha>0, \beta \neq 0)$. This functional form represents increasing or decreasing demand for different values of the parameter $\beta$.

We have,

$$
\begin{aligned}
& \frac{d R(t)}{d t}=\alpha \beta e^{(\beta-1) t} \\
& \frac{d^{2} R(t)}{d t^{2}}=\alpha \beta(\beta-1) e^{(\beta-2) t}
\end{aligned}
$$

Then the following possible cases arise: 
1. For $0<\beta<1, \frac{d R(t)}{d t}>0$ and $\frac{d^{2} R(t)}{d t^{2}}<0$, the demand increases with time at a decreasing rate. We may call it retarded growth in demand. This usually happens when some new products launched in the market. For such products, the demand rate goes on increasing gradually approached a saturation level.

2. For $1<\beta<2$ and $\beta>2, \frac{d R(t)}{d t}>0$ and $\frac{d^{2} R(t)}{d t^{2}}>0$, which implies that the demand will go on increasing with time at an increasing rate. We may call it accelerated growth in demand which is seen in the case of spare parts of newly introduced state-of-the art aircrafts, computers etc. This also happens to the seasonal products like winter cosmetics, winter vegetables, fruits towards the beginning of the season.

3. For $\beta<0, \frac{d R(t)}{d t}<0$ and $\frac{d^{2} R(t)}{d t^{2}}<0$, which implies that the demand will go on decreasing with time at a decreasing rate. This type of demand rate undergoes an accelerated decline in demand. This usually happens in the spare parts of the obsolete aircrafts, computer chips of high technology products, which is being substituted by another. This is also applicable to the seasonal products towards the end of the season.

\section{References}

1. R. Gupta and P. Vrat, Opsearch 23, 19 (1986).

2. R. C. Baker and T. L. Urban, Journal of the Operational Research Society 39, 823 (1988).

3. E. Ritchie, Journal of the Operational Research Society 35, 949 (1984).

4. W. A Donaldson, Operational Research Quarterly 28, 663 (1984). doi:10.2307/3008916

5. E. A. Silver and H. C. Meal, Production and Inventory Management 14, 64 (1973).

6. M. Deb and K. S. Chaudhuri, Journal of the Operational Research Society 38, 459 (1987).

7. U. Dave, Journal of the Operational Research Society 40, 412 (1989).

8. S. K. Goyal, D. Morin, and F. Nebebe, Journal of the Operational Research Society 43, 1173 (1992).

9. A. Goswami and K. S. Chaudhuri, Journal of the Operational Research Society 42, 1105 (1991).

10. U. Dave and L. K. Patel, Journal of the Operational Research Society 32, 137 (1981).

11. R. S. Sachan, Journal of the Operational Research Society 35, 1013 (1984).

12. V. Aggarwal and H. Bahari-Kashani, IIE Transaction 23, 185 (1991). doi:10.1080/07408179108963853

13. H. M. Wee, Computational Operational Research 22, 345 (1995). doi:10.1016/0305-0548(94)E0005-R

14. F. Raffat, P. M. Wolfe, and H. K. Eldin, Computers and Industrial Engineering 20, 89 (1991). doi:10.1016/0360-8352(91)90043-6

15. K. L. Mak, Computers and Industrial Engineering 6, 309 (1982). doi:10.1016/0360-8352(82)90009-2

16. S. K. Ghosh, S. K. Goyal, and K. S. Chaudhuri, International Journal of System Science 37, 1003 (2006). doi:10.1080/00207720600813095

17. Z. T. Balkhi and L. Benkeherouf, European Journal of Operational Research 92, 302 (1996). doi:10.1016/0377-2217(95)00148-4

18. H. M. Wee and S. T. Law, Computers \& Operations Research, 26, 545 (1999). doi:10.1016/S0305-0548(98)00078-1 
19. C. T. Su, C. W. Lin, and C. H. Tsai, Journal of the Operational Research Society of India 36, 95 (1999).

20. L. Y. Ouyang, K. S. Wu, and M. C. Cheng, Yugoslav Journal of Operations Research 15, 277 (2005). doi:10.2298/YJOR0502277O

21. R. H. Hollter and K. L. Mak, International Journal of Production Research 21, 813 (1983). doi:10.1080/00207548308942414

22. A. K. Bhunia and M. Maiti, Journal of the Operational Research Society 48, 221 (1997).

23. A. Goswami and K. S. Chaudhuri, International Journal of System Science 22, 181 (1991). doi:10.1080/00207729108910598 